\title{
Prácticas y categorías de parentesco en Jasimaná, noroeste andino de Argentina
}

\section{Practices and categories of kinship in Jasimaná, north western andean Argentina}

\author{
Daniela Salvucci ${ }^{1}$
}

\section{Resumen}

Pese a la difusión de la ideología genealógica española y del modelo católico de unión y descendencia legítima que se dio a partir de la colonización, en la Argentina andina existieron y siguen existiendo "culturas" indígenas de "familia" y "parentesco". El objetivo del texto es analizar, desde una perspectiva de la antropología cultural, las categorías émicas y las prácticas del parentesco en el altiplano de Jasimaná (provincia de Salta, Noroeste de Argentina, NOA). El método etnográfico y el estudio de caso, a partir de reconstrucciones gráficas de redes familiares, nos permitirá enfocar las dinámicas de los grupos domésticos y sacar a la luz las características culturales locales del parentesco en relación a la inserción histórico-cultural de Jasimaná en el NOA y en la macro área andina.

Palabras claves: parentesco andino, grupos domésticos, Noroeste Argentino (NOA).

\begin{abstract}
During the Spanish colonization, the genealogical ideology and the Catholic model of legitimate marriage and descent have spread in America. Nonetheless, indigenous "cultures" of family and kinship have lasted and they still exist in Andean Argentina. The text aims to analyse emic categories and practices of kinship people performe in the highland of Jasimaná (province of Salta, NWA-North Western Argentina), from the perspective of cultural anthropology. Ethnographic method, case study and graphic reconstruccions of family networks allow us to focus on the dynamics of the local domestic groups to point out their cultures of kinship, taking under consideration that the highland is part of a wider historical and cultural context, such as the North Western of Argentina and the Andean macro area.
\end{abstract}

Keywords: Andean Kinship, domestic groups, NWA - North Western Argentina.

Recibido: 12 abril 2016. Aceptado: 6 enero 2017

1 Antropóloga cultural, Investigadora de la Libre Universidad de Bolzano. Dirección: viale Ratisbona, 16 - 39042 Bressanone, Bolzano, ITALIA. Email: salvuccidana@gmail.com 


\section{Culturas del parentesco}

En las décadas de los 1970 y 1980 varios antropólogos, sobre todo de la escuela anglosajona, se comprometieron con deconstruir la noción eurocéntrica de parentesco (kinship). A fin de eludir dicha palabra-concepto, Carsten (2000) creó la expresión culture of relatedness, que podría traducirse como "culturas del estar en relación/en relación de parentesco", jugando con el doble sentido en el idioma inglés. Según la autora ya no se trata de estudiar un sistema formal de reglas de descendencia y alianza, sino enfocar las experiencias culturales locales de las relaciones. Si bien otros autores siguieron utilizando el término "parentesco", igual subrayaron la necesidad de extender su posible definición, trasformando este concepto en una categoría abierta a las múltiples prácticas locales y a las diversas percepciones, para abarcar diferentes "culturas del parentesco" (Piasere y Solinas, 1998).

Desde la perspectiva culturalista, lo que se percibe y practica como "parentesco", "familia" y "hogar" puede ser muy diferente de un contexto a otro, ya que se trata de construcciones culturales locales que no radican siempre en la separación entre "natura" (lo biológico, el parentesco consanguíneo) y "cultura" (lo simbólico, político y social). Una separación, en cambio, que fundamenta tanto el concepto occidental euroamericano de parentesco (Schneider, 1968) como la categoría "parentesco" utilizada por los antropólogos. Según Stone (2001), una nueva antropología del parentesco implicaría una operación de traducción para entender qué es "parentesco" para los actores locales y qué para el antropólogo. No se trata, por lo tanto, de negar la existencia del "parentesco", como lo proponen algunas críticas más radicales (Schneider, 1984), sino reformular su sentido y los enfoques para estudiarlo, prestando atención a las teorías, a las prácticas materiales y simbólicas locales.

En nuestro caso, los criterios de consanguinidad en tanto "sustancia biogenética compartida" y de afinidad como alianza jurídica, que sustentan la noción euroamericana de parentesco "by blood" (por sangre) y "by law" (por ley) según Schneider (1968), no son necesariamente los que fundamentan las relaciones parentales de los habitantes de Jasimaná.
En el altiplano andino, muchos hijos son "criados" y pueden ser nietos de sus "padres", o hasta no tener lazo alguno de consanguinidad con ellos. Además, según la teoría clásica de la alianza (Lévi-Strauss, 1949) la unión de la pareja es un intercambio que permite la construcción de grupos sociales, pero a nivel local la relación de alianza puede ser inexistente. En Jasimaná la mayoría de las parejas se junta de manera informal y muchas lo hacen de forma transitoria aunque reproductiva. Los hombres-padres circulan de un hogar a otro, mientras que las mujeresmadres solteras y los abuelos se "hacen cargo" de los niños, que pueden ser llamados "hijos del viento" cuando se desconoce, o sea no se "hace reconocer", al padre. Los actores sociales locales, de hecho, producen sus relaciones y utilizan sus propias categorías para expresarlas. Por lo tanto, ¿de qué categorías se trata? ¿Qué relaciones expresan y a cuáles prácticas están asociadas?

En este ensayo conceptualizamos las "culturas del parentesco" como conjuntos de categorías y prácticas de relaciones cuya experiencia se vive a nivel local. Sin embargo, dicho nivel se inserta en un contexto histórico-cultural de difusión de variantes de alcance regional y macrorregional, lo del Noroeste de Argentina (NOA) y del área andina. En el apartado siguiente se propone un breve recorrido de los estudios de parentesco en la macro área andina. A continuación, se expondrá el marco teórico interpretativista de la investigación y se presentará el altiplano de Jasimaná, donde se realizó el trabajo de campo etnográfico. En el quinto apartado se muestra un estudio de caso basado en la reconstrucción gráfica de dos "redes familiares" de pastoras del altiplano, dońa Dina y sus hijas por un lado, dońa Ana y su hija Marta, por el otro. El estudio de caso será utilizado como punto de partida para analizar, en los dos apartados siguientes, las prácticas y las categorías locales asociadas a la "cocina", a la "casa", a la "hacienda" (rebaños), subrayando la centralidad de las relaciones basadas en "ser familia", "criar" y tener "hijos de soltera". Por fin, en el último apartado se sacarán conclusiones relativas al ámbito cultural local del parentesco, subrayando, por una parte, la operatividad en Jasimaná de categorías y prácticas difundidas a lo largo de la macro área andina. Por otra, se pondrá de relieve que el ámbito doméstico 
y familiar local incluye no solo a las personas, sino también los animales y otras entidades, como los Santos de la casa y las "almas" de los antepasados.

\section{Parentesco en el área andina}

El Noroeste Argentino (las actuales provincias argentinas de Jujuy, Salta, Catamarca, Tucumán, La Rioja y Santiago del Estero) constituyó la periferia sur del Tawantinsuyo (Lorandi, 1980), el Imperio inca, para luego conformar el Tucumán Colonial (junto a la actual provincia de Córdoba) en el virreinato del Perú. Esta región forma parte del área andina meridional (Lorandi, 1997), junto con el norte de Chile y la Bolivia meridional, en donde se dieron largos procesos de intercambio económico y cultural, además de importantes desplazamientos poblacionales y fenómenos de mestizaje y afromestizaje (Lorandi, 1980, 1990-92; Mata de López, 2005; Rodríguez, Lorandi, 2005).

Antes de la anexión al Tawantinsuyo, el sector valleserrano del NOA, donde se encuentra el altiplano de Jasimaná, estaba poblado por diferentes grupos diaguitas, de idioma kakan, a los que se agregaron conjuntos de mitmaqkunas. Éstos eran colonos de idioma quechua desplazados por los incas desde el sur del territorio de Cusco (Lorandi, 1997, p. 219) y desde la llanura tucumano-santiagueña (Lorandi, 1980) para controlar la región diaguita. La resistencia de estos grupos, llamados "indios calchaquíes" por los españoles, desde el nombre del cacique diaguita que encabezó el primer levantamiento en 1562, al que se sumaron dos levantamientos más, impidió la colonización espańola del área de los valles y cerros calchaquíes hasta el 1667 (Lorandi, 1997a). A finales del siglo XVII y a lo largo del XVIII, este sector vallemontano del Tucumán Colonial se incorporó a los circuitos comerciales macrorregionales (Assadourian, 1982; Mata de López, 2005), atrayendo grupos migrantes de "indios forasteros" (SánchezAlbornoz, 1978) quechua y aymara del Alto Perú (Lorandi, 1997; Mata de López, 2005) y a grupos atacameños de idioma kunza (Hidalgo, 1985). Además, pese a los actuales confines nacionales entre Bolivia, Chile y Argentina, esta macro área sigue siendo una región de intercambio y circulación de personas, objetos y prácticas.
Para contextualizar las culturas locales del parentesco, por lo tanto, hay que tomar en cuenta la inserción histórico-cultural de Jasimaná en el NOA y en el área andina meridional. Un breve recorrido a lo largo de la literatura antropológica sobre "parentesco andino” nos permitirá, además, subrayar los cambios de perspectiva teórica y metodológica que se dieron en este ámbito de estudios.

El interés de los diferentes autores que describieron los "sistemas" de parentesco y familia de las poblaciones andinas quechua (Castro Pozo, 1946; Mishkin, 1946) y aymara (Tschopick, 1946) contemporáneas en el segundo volumen del Handbook of South American Indians editado por Steward (1946), en el marco estructural-funcionalista de la década de los cuarenta, se concentraba en las teorías de la descendencia. Ésta podía ser de tipo bilateral (Mishkin, 1946) o unilineal, generalmente asociada a las familias extensas patrilineales y patrilocales o al ayllu de origen incaico, considerado un grupo corporado tanto de descendencia por algunos como de residencia por otros (Ossio, 1982; Sendón, 2005).

Si bien se identificaron grupos corporados de descendencia patrilineal, como los llamados castas (Vásquez y Homberg, 1966), ya en la década de los setenta los antropólogos ponen de relieve la "bilateralidad" del parentesco en los Andes. Con este título, en su introducción al volumen editado por Mayer y Bolton (1980 [1977]) sobre parentesco y matrimonio en los Andes, Lambert (1980 [1977]) resume los hallazgos de varios trabajos sobre el tema, destacando dos características generales del parentesco andino: la estructura cognática y bilateral, según el modelo del kindred de Freeman (1961), en relación a la descendencia, ${ }^{2}$ y la centralidad de la pareja conyugal con respecto a la alianza.

2 Sin embargo, el ensayo de Belote y Belote (1980 [1977]) del mismo volumen puso en evidencia rasgos de descendencia paralela en las comunidades saraguros de Ecuador con respecto a la teoría local de la fecundación y a las prácticas de herencia de las devociones a los santos, rasgos que serán subrayados también por Arnold y otros (1998) en relación a la herencia de fluidos corporales, de bienes y derechos de los pobladores de Qaqachaka en Bolivia. 
Los estudios de las décadas de los setenta y ochenta, de hecho, se focalizan en el grupo doméstico asociado a la familia conyugal en tanto que unidad-base del sistema económico y ecológico andino (Brush, 1977), influidas por las teorías de la economía familiar campesina (Wolf, 1966; De la Peńa, 2001) y, sobre todo, del funcionalismo ecológico (Murra, 1975; Brush, 1977; Sendon, 1985; Rabey, Merlino y Gonzalez, 1986). Tanto las comunidades como los grupos domésticos pueden manejar el control de diferentes "pisos ecológicos verticales" (Murra, 1975) y la organización autónoma de la producción y del consumo. Por una parte se subraya el papel central de los lazos de parentesco, inclusive del parentesco espiritual, en la organización social y ecológica comunitaria (Harris, 1985; Mayer, 2004, Isbell, 2005 [1978]). Por la otra, se considera la pareja conyugal como el eje de este sistema, tanto a nivel ecológico como simbólico, juntando así el funcionalismo ecológico y la teoría estructuralista de la "complementariedad dual de género" andina, pese al reconocimiento de cierta asimetría en dicha ideología, tanto local como académica, de la complementariedad (Platt, 1978).

En la década de los noventa, las teorías culturalistas y deconstrucionistas de la antropología del parentesco empujan hacia estudios que abordan las prácticas diarias y los significados locales de parentesco, hogar y género en los Andes también (Arnold, 1997, 1998). Criticando el concepto de "complementariedad dual de género" y la centralidad de la pareja andina en las teorías funcionalista y estructuralista, los estudios de Spedding (1998) ponen de relieve el alto porcentaje y la importancia de las madres solteras en comunidades de los Andes bolivianos. Arnold (1998a) subraya además las muchas características de "matrifocalidad" del parentesco andino, como la centralidad de la casa y de la despensa asociada a la mujer, que los investigadores estructural-funcionalistas pasaron por alto enfatizando descendencia patrilineal y residencia patrilocal.

En relación con la despensa y la cocina, Weismantel $(1988,1998)$ señala la importancia del "dar de comer" y de la "circulación de comida" en tanto acciones que producen relaciones de parentesco en Zumbagua, una comunidad andina de Ecuador. Según la autora, es la comida y no la "sangre" el lazo que une (Weismantel, 1988, p. 171). Con respecto a los "hijos", por ejemplo, lo que el antropólogo podría interpretar como "parentesco" no se basa ni en la consanguinidad ni en la adopción jurídico-social, ya que es la práctica del dar de comer lo que "hace materialmente" al hijo. La categoría utilizada por los actores locales es "viñachina", una palabra quechua que significa "criar" y que se refiere no solo a las personas, sino también a las plantas y a los animales criados (Weismantel, 1998, p. 91). La misma palabra aymara, "uywaña" (criar), expresa el afecto, la ternura y el carińo del cuidado y del criar, como lo sugiere Palacio Ríos (1982). Este concepto se asocia a la casa, ya que los espíritus de la casa se llaman "uywiri", es decir, "los que crían". La categoría "casa" conlleva prácticas y percepciones culturales específicas. Para los pastores de altura del Noroeste andino de Argentina, "casa" implica una dinámica de movimiento entre la "casa principal", la "casa en el pueblo" y los varios "puestos" o "estancias" como se les llama en la puna jujeńa (Tomasi, 2013). La casa incluye recintos techados y sin techar: se trata de las piezas para las personas y los corrales, que los pobladores definen como la "casa de la hacienda", es decir, de los rebaños (Delfino, 2001; Göbel, 2001, Tomasi, 2013). En este contexto, las prácticas del "criar" y la relación de afecto y cariño se refieren también a los animales que, como lo han señalado Bugallo y Tomasi (2012, p. 212), son parte del grupo doméstico.

Ya que el altiplano de Jasimaná pertenece a dicha geografía histórico-cultural andina, ¿̨cuáles prácticas y percepciones los pobladores de Jasimaná refieren a la casa y a la cocina? ¿Qué papel juega la "hacienda" en la teoría local del grupo doméstico? Por fin, ¿a qué dinámicas de relación a nivel local se refieren las categorías "clásicas" de la antropología, como "parentesco", "familia", "hogar"?

\section{Interpretación y problemas de traducción}

Según Geertz (1973) la meta de la investigación antropológica es entender el sentido de las acciones realizadas por personas específicas, en un lugar y tiempo definidos. No se trata de demostrar una teoría general, sino profundizar y utilizar ideas teóricas existentes para interpretar un conjunto de significados 
que, pese a su perspectiva semiótica, este autor concibe como incorporados en las acciones sociales. Las interpretaciones, además, tienen que orientarse hacia los "actores sociales", hacia sus sentidos y "categorías émicas", 3 definidas "desde adentro" del universo cultural local. Participar en la acción y en las prácticas diarias, tanto laborales como festivas y rituales, permite acercarse a los significados de los actores sociales.

Los datos cualitativos analizados a continuación han sido producidos en este marco metodológico interpretativista, por medio del trabajo de campo y de una etnografía fundada en la observación participante, en la interpretación de las prácticas y en la traducción de las categorías émicas a partir de "categorías éticas" antropológicas como "parentesco", "grupo doméstico" y "red familiar". Como base para la traducción se produjeron reconstrucciones gráficas de redes familiares según la forma del kindred bilateral y ego-centrado (Freeman, 1961) a partir de la información brindada por un ego-informante. También se reconstruyeron "redes integradas", por medio de la técnica del "desplazamiento del ego" (Sinibaldi, 2008; Salvucci, 2010), juntando la información brindada por otro ego (ego 2) perteneciente a la misma red familiar.

En las redes se marcaron las formas de los "grupos domésticos" (también definidos en este texto como "hogares" o "unidades domésticas"), usando la tipología elaborada por Peter Laslett $(1972),{ }^{4}$ aun-

3 Geertz utiliza la distinción entre "émico” y "ético” creada por el lingüista Pike (1954) y elaborada en la antropología por M. Harris (1968). Estos conceptos derivan de phonemic y phonetic: "fonémica" es la perspectiva centrada en el estudio de las unidades significativas para el hablante, mientras que "fonético" es el estudio de los sonidos del hablante como se les apercibe desde el exterior.

4 Según Laslett, la unidad doméstica (household) corresponde a un grupo de personas que viven juntas ("bajo un mismo techo") y que tienen lazos de parentesco entre sí, sobreponiendo de esta manera los dos conceptos de "familia" y "hogar" (Yanagisako, 1979). De acuerdo con dicha tipología hay hogares nucleares o conyugales simples, conformados por la pareja de los padres con (o sin) sus hijos, o por un viudo o viuda con los hijos; hogares extensos, es decir, una familia nuclear con otros parientes (por ejemplo uno de los padres de la pareja o los nietos); hogares multinucleares, que juntan a más que tomando en cuenta los comentarios críticos de Yanagisako (1979) a dicha tipología. Según esta autora, para estudiar los "grupos domésticos" cabe considerar las actividades de grupos concretos y sus prácticas de relaciones en la vida diaria, además de las percepciones culturales locales de "familia", "ámbito doméstico" y "casa”, entre otras.

El estudio de caso presentado nos permitirá ver que las categorías antropológicas no siempre logran explicar las situaciones concretas y que estos conceptos solo tienen sentido en una perspectiva de traducción, como punto de partida para acercarnos a comprender las relaciones locales y las categorías émicas. Para dar cuenta de situaciones concretas, se eligió la modalidad del estudio de caso. Los datos etnográficos utilizados han sido recolectados durante dos trabajos de campo realizados en 2011 y 2015 en Río Grande y El Arremo de Jasimaná. ${ }^{5}$

\section{Jasimaná}

Jasimaná (municipalidad de Angastaco, departamento de San Carlos) es un vasto territorio que se encuentra entre los 3000 y 3800 msnm aproximadamente, en el suroeste de la provincia de Salta, en el sector valleserrano de la cordillera andina centro-meridional.

Los habitantes (con la excepción de los pobladores del paraje de Los Cardones) conforman la Comunidad Indígena Diaguita-Calchaquí. La mayoría de ellos son pastores de llamas, cabras y ovejas, además de ser, al mismo tiempo, artesanos, comerciantes y trabajadores asalariados. Hoy en día, gracias a la lucha de la comunidad indígena, la gestión del territorio es comunitaria: los miembros eligen un cacique y una comisión y las decisiones se toman en asamblea. El uso de las tierras se maneja según el principio del "respeto", es decir, respetando los confines de los lotes de pastizales de cada familia.

familias conyugales; hogares unipersonales; el complejo residencial familiar, que junta todos los que comparten un mismo complejo de habitaciones y casas.

5 En Río Grande y El Arremo he frecuentado diferentes grupos domésticos, participando en las tareas diarias, en las fiestas familiares, en los rituales, y realizando 15 entrevistas individuales semiestructuradas sobre historia de vida e historia de familia para reconstruir las redes familiares egocentradas. 
Las actuales aldeas de Río Grande, El Arremo y Pampallana surgieron entre fines de los setenta y la década de los ochenta alrededor de las escuelas primarias construidas en aquel entonces. ${ }^{6}$ En cada una de las aldeas residen aproximadamente 250 habitantes, según los datos de los Agentes Sanitarios (com. pers.), la mitad de los cuales son menores de edad.

En los ańos noventa se realizó el camino de tierra que permite subir en camioneta, en aproximadamente cuatro horas de viaje, desde Angastaco hasta Río Grande y El Arremo (y desde ahí hasta Pampallana). Cuando todavía no había camino, según los relatos recolectados, los varones de cada grupo doméstico realizaban largos viajes con caravanas de burros de carga para intercambiar o vender los productos del altiplano (carne, cueros, yuyos del monte, lana e hilados) y comprar mercadería, yendo hacia los pueblos de los Valles Calchaquíes catamarqueños y salteños, como Santa María, Cafayate o Angastaco, para abastecerse de trigo, maíz, verduras, frutas, vino y otros bienes.

Actualmente, los grupos domésticos y sus miembros no residen de forma fija en las aldeas, sino que se desplazan entre diferentes viviendas, según el patrón de asentamiento determinado por los circuitos de la trashumancia, como en varias comunidades de pastores de altura de la macro área andina y del Noroeste de Argentina (Rabey, Merlino y Gonzalez, 1986; Göbel, 2002, Tomasi, 2013). Muchos comuneros viven en la aldea para que los hijos puedan ir a la escuela, pero su "casa principal" (también llamada “casa de campo") está en el altiplano, aislada. ${ }^{7}$ Aquí

6 Cada una de las aldeas cuenta con escuela primaria (en Río Grande y El Arremo hay también un secundario de temporada), una capilla católica y un puesto de salud con un enfermero residente y un agente sanitario. Cuando se realizaron los trabajos de campo, si bien las escuelas, los puestos de salud y unas cuantas viviendas privadas tenían paneles solares o generadores a gasolina, en las aldeas no había red eléctrica pública que funcionara, tampoco conexión telefónica, ni internet.

7 En la casa de campo, por lo general, las paredes de las varias piezas son de piedras y barro; el cielo raso de ramas con ejes de madera de cardón (Echinopsis atacamensis); el techo a dos aguas es de paja o torta de barro; la puerta de tablas de madera de cardón. La cocina es una pieza con fogón y chimenea. En la aldea, las casas guardan una estructura parecida, compuestas por pie- se encuentran los corrales para la "hacienda" (como son llamados los rebaños) de ovejas y cabras. Las llamas no son acorraladas a diario, sino que tienen sus dormitorios en el campo. Por lo general, los pastores se desplazan según la temporada y los circuitos de la trashumancia, entre la casa principal y el "puesto", una casa más pequeña, también con corrales, en la tierras altas del altiplano. ${ }^{8}$

La modalidad de trabajo con los rebaños es el pastoreo extensivo, y los animales que han sido acorralados por la noche son "despachados" por la mañana para que se alejen lo más posible en busca de pasto. Por la tarde, mientras que las cabras pueden regresar solas al corral, las ovejas tienen que ser empujadas, lo que hacen los pastores con la ayuda de los perros $\mathrm{y}$ a veces de una honda a lazo con la cual echan piedras para estimular a los animales a volver. Por lo general, un pastor o una pastora principal se ocupa de la hacienda familiar que junta a las reses de los diversos miembros del grupo doméstico y de la familia. Hasta los miembros emigrados al valle o a la ciudad pueden seguir teniendo sus animales en la hacienda familiar.

El cuidado de la hacienda es considerado una tarea femenina, mientras que los varones deberían trabajar en la agricultura o criar ganado vacuno y mular. Sin embargo, son muchos los hombres que se ocupan de los rebaños, principalmente de las llamas. Otros viajan en camioneta para abastecer los almacenes de las aldeas, de su propiedad, trayendo mercadería desde las ciudades del valle, mientras que muchos changos (jóvenes) emigran temporáneamente, pero también de forma permanente, a los pueblos del valle, a la ciudad de Salta o a las regiones del sur para trabajar como mano de obra en construcción o en fincas. También las chicas emigran, generalmente de forma temporal, por unos meses y hasta años, trabajando como empleadas domésticas en Salta o en

zas independientes, aunque, por lo general, son hechas de adobe, con techo de chapa.

8 Los pastores se desplazan con sus rebaños entre las tierras bajas del altiplano, donde los animales aprovechan los pastos frescos que crecen después de las lluvias de enero y febrero, y las tierras altas donde, en la temporada seca y fría, se puede aprovechar la vegetación permanente de las altitudes mayores cuando ya no queda pasto abajo. 
Cafayate, aunque muchas de ellas regresan al altiplano al quedar embarazadas, para criar a su guagua (o wawa, bebé en quechua) en la casa de sus padres o de su madre. La mayoría de los pastores y pastoras, además, trabajan la lana que producen y venden sus hilados y tejidos en los mercados de artesanía de los pueblos del valle, sobre todo en Cafayate, y en varios festivales de artesanía de la región.

Con respecto a los patrones migratorios de las comunidades andinas del NOA, muchos autores han subrayado que la migración estacional constituye una estrategia (Rabay et al., 1986) de los grupos domésticos "pluriactivos" del altiplano (Bugallo, 2014), basados en la "multiocupación" (Parodi, Benedetti, 2016, p. 32), para tener un ingreso monetario. Las migraciones estacionales pueden generar "ciclos" hasta de "año redondo" que llevan los migrantes, principalmente los varones, a seguir las diferentes cosechas ${ }^{9}$ de la región y de otras áreas del país (Reboratti, 1976, p. 235). Con respecto a las migraciones permanentes, los habitantes de Jasimaná se desplazan sobre todo hacia los pueblos del valle, como San Carlos y Cafayate, pero también hacia la ciudad de Salta. En muchos casos la migración permanente no quiebra los lazos con el altiplano y con la familia que ahí se queda, creando un ámbito "rural-urbano" de relaciones activas, como ocurre también en otras comunidades de la macro área andina (De la Cadena, 1988; Mayer, 2004; Malengreau, 2007; Carrasco y González, 2014).

\section{Redes familiares y grupos domésticos: un estudio de caso}

El dibujo que se presenta a continuación (Figura1) muestra las redes familiares de dos primas hermanas que se dibujó por separado durante el trabajo de campo en Río Grande y que luego se unió a partir de la referencia al lazo de parentesco hecha por las

9 Las entrevistas realizadas en Jasimaná ponen de relieve la participación de los varones, pero también de las mujeres, en las cosechas de tabaco en el valle de Lerma (Salta), de cebolla y ají en el Valle Calchaquí (Salta), de cítricos en la llanura tucumana (Tucumán), la vendimia en las provincias de Salta, La Rioja y Mendoza, y, hasta hace unos años, la zafra, es decir, la cosecha de la caña de azúcar en las provincias de Salta y Tucumán. mismas mujeres. La red de dońa Dina,${ }^{10}$ que ocupa la parte derecha de la Figura 1, se realizó por medio de la técnica del "desplazamiento del ego", juntando la información brindada por dońa Dina (ego 1) y luego por su hija Rosa (ego 2). La segunda red, a la izquierda en el gráfico, se dibujó a partir de la entrevista con Marta (ego 3), hija de doña Ana, una prima hermana de dońa Dina.

Doña Dina (ego 1, a la derecha en la Figura 1, página siguiente) es una mujer de aproximadamente 60 años, viuda desde hace poco, que trabaja de empleada doméstica en la escuela de la aldea de Río Grande. Sus hermanos y hermanas dejaron el altiplano de forma permanente desde hace mucho tiempo, así como la mayoría de sus nueve hijos, quienes emigraron a Cafayate, a Salta y a la provincia de Buenos Aires. Solo dos de sus hijas se quedaron en Jasimaná: Rosa y Adela, ambas madres solteras de aproximadamente 30 años. Doña Dina vive en su casa en la aldea, junto con su hija menor, Adela, las dos hijas de Adela (respectivamente de 15 y 9 años) y el hijo mayor de Rosa, un chico ya adolescente que ha sido criado por dońa Dina. En la Figura 1 señalé este grupo doméstico por medio de un contorno rectangular negro (el segundo que se encuentra desde la derecha), aunque Adela no vive siempre en casa de la aldea con su mamá y sus hijas, sino en la casa de campo que pertenecía a su abuelo (el padre de dońa Dina) que se encuentra a una hora de camino de la aldea, subiendo hacia los cerros, en un lugar llamado Cerro C. Aquí, Adela se ocupa de los rebańos de cabras y ovejas. ${ }^{11}$ El fin de semana, sus hijas y su sobrino suben a la casa principal para ayudarla con los animales. A veces doña Dina también sube o se queda ella misma en la casa de campo cuando la escuela está cerrada, mientras que Adela regresa a la aldea. Durante la temporada seca, el abuelo de Adela solía trasladar los animales desde la casa principal hacia el puesto, en los cerros. Este puesto se derrumbó hace varios años y Adela y su mamá ya no lo usan. En ocasiones especiales, como el rito del "pago a la Tierra", cuando se hace

10 Todos los nombres han sido modificados para respetar el anonimato de los amigos informantes. De los nombres de los lugares que pertenecen a las familias -por derechos sobre esas tierras-, solo se escribió la inicial.

11 Adela no tiene llamas, ya que en el arroyo de los alrededores, donde toman agua los animales, existe el unco, un parásito que mata a estos animales. 


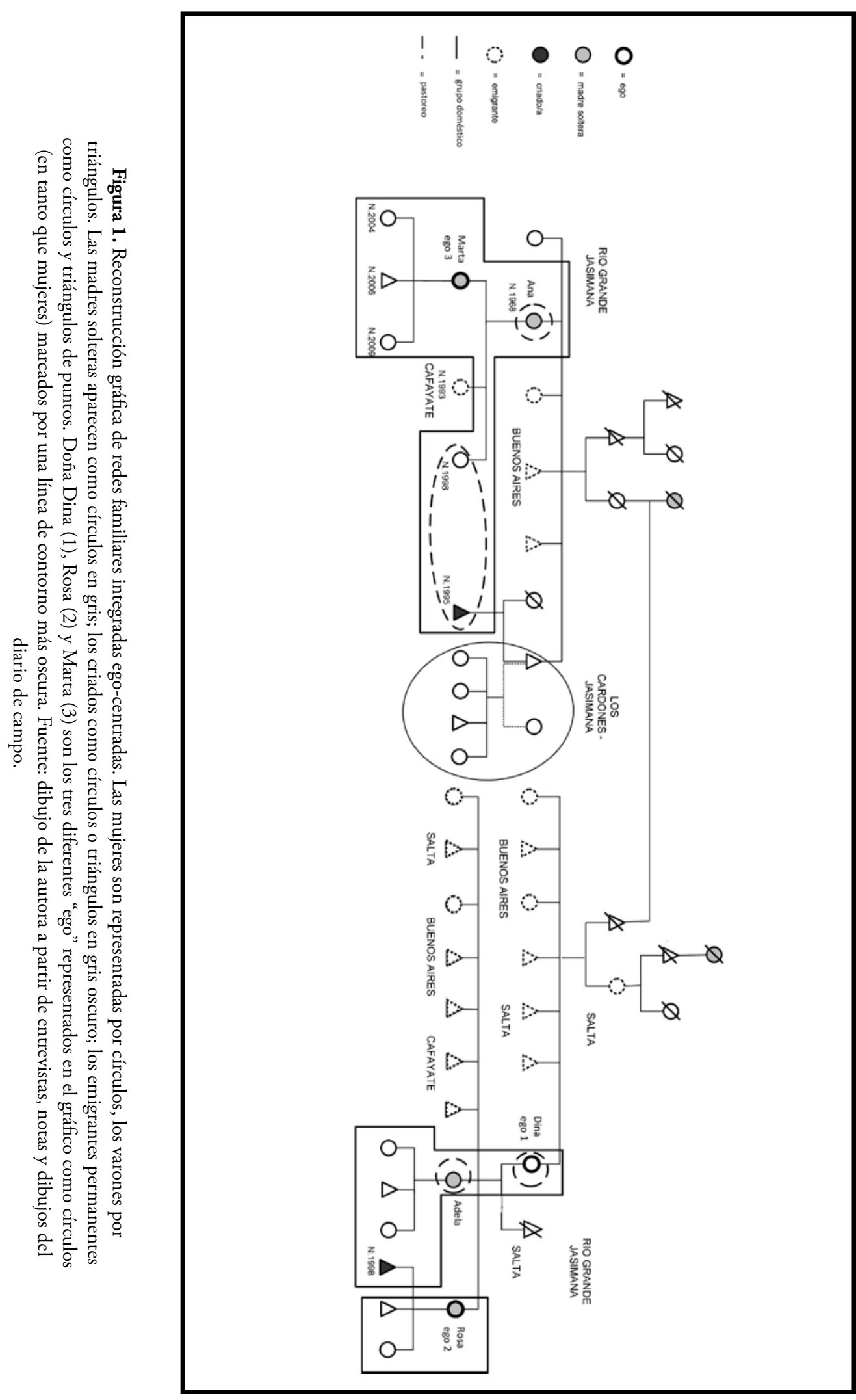


una ofrenda grande a la Pachamama-Santa Tierra, o para las "señaladas", cuando se marcan los animales jóvenes de la hacienda familiar con cortes en las orejas, tanto doña Dina con las hijas de Adela y el nieto criado como Rosa y sus otros dos hijos se reúnen en la casa principal en Cerro C. Por lo general, Rosa no se ocupa de los rebańos pero ayuda a su mamá y hermana en estas ocasiones de trabajo-ritual y también durante la esquila de las ovejas. Además, Rosa y sus dos hijos menores tienen sus animales en la hacienda familiar cuidada por Adela. En la aldea, Rosa y sus dos hijos menores viven en una casa aparte (este grupo doméstico ha sido señalado en línea de contorno rectangular negro en la Figura 1: es el primer grupo que se encuentra desde la derecha). Rosa viaja frecuentemente a Cafayate y San Carlos, en el valle, para vender los tejidos que tanto ella como su hermana Adela producen. Durante la entrevista, Rosa cuenta de su experiencia migratoria en la ciudad de Salta, a donde se fue a vivir a los 14 ańos en la casa de una familia burguesa trabajando de empleada doméstica. Luego de haber quedado embarazada, Rosa volvió a Jasimaná. Su mamá también vivió por un tiempo en Tolombón, en el valle, a partir de los 12 años, cuando sus padres la mandaron a la casa de una familia conocida para trabajar en las labores domésticas y poder así, al mismo tiempo, asistir a la escuela, ya que en aquella época no la había en el altiplano.

Con respecto a la segunda red familiar de la Figura 1, ésta se dibujó a partir de Marta, una joven de 28 años (en 2015), madre soltera de tres hijos (una chica de 14 ańos, un chico de 9 y una nena de 6) que vive en la aldea de Río Grande con ellos, una hermana menor y un hermano de crianza, el hijo de un hermano de su mamá Ana, criado por esta última. El papá del chico, después de enviudar, se unió con otra mujer y se mudó a Los Cardones, un paraje a tres horas de camino de Río Grande, formando otro grupo doméstico (en línea de contorno circular y negra en la Figura 1). La otra hermana de Marta vive en Cafayate, donde estudia albergada en el colegio secundario. Después de haber acabado la escuela, Marta emigró por seis meses a Cafayate, pero cuando quedó embarazada volvió a Jasimaná. Marta, hija a su vez de madre soltera, "reconoce" a su padre natural, en el sentido de que los dos se conocen, saben de la relación que les une y se "respetan", por ejemplo saludándose cuando se encuentran.
Doña Ana, la madre de Marta, una señora de 55 ańos, se ocupa de la hacienda familiar compuesta por rebaños de cabras y ovejas y vive en la casa de campo del grupo doméstico, a una hora de camino de la aldea, en un lugar llamado Ciénaga G., cuyos pastizales lindan con los de Cerro C. de Adela y doña Dina.

Como se muestra en la Figura 1, la mamá de dońa Ana y el papá de dońa Dina eran hermanos, hijos de madre soltera de la cual heredaron los derechos de uso de los pastizales de Ciénega G. por un lado, y de Cerro C. por el otro. Tanto Marta como Adela (primas segundas) hacen referencia a esta bisabuela madre soltera que les dejó derechos sobre las tierras.

Doña Ana, si bien vive en la casa principal, cuando baja a la aldea se queda en la casa de su hija Marta. Por el fin de semana, su hija menor, su sobrino y a veces sus nietos suben a Ciénega G. para ayudarla con los animales o para quedarse con la hacienda, permitiéndole así bajar a la aldea o a los pueblos del valle. Además, en ocasión del pago a la Tierra, de las señaladas y de la esquila de las ovejas, Marta también sube a la casa principal, donde se reúne todo el grupo doméstico.

\section{“Cocina", "casa" y "hacienda"}

En Jasimaná se hace referencia a la cocina para indicar a los que viven juntos: la categoría émica "tener la cocina aparte" significa que un grupo de personas tienen su propia cocina, es decir, "comparten" dicha cocina y estan aparte de otros grupos. Pero una cocina "aparte" conlleva muchas prácticas diferentes: es posible tener una cocina "aparte" en la casa en la aldea, pero compartir la cocina en la casa de campo.

En el caso de doña Dina y su hija Adela, las dos mujeres comparten la cocina tanto en la casa principal como en la casa de la aldea, mientras que Rosa, la otra hija de dońa Dina, tiene su casa y su cocina aparte en la aldea, aunque comparte la cocina de la casa de campo con su madre y su hermana. Marta y su madre, dońa Ana, comparten la cocina tanto en la casa de la aldea como en la casa de campo. Según la tipología de Laslett (1972), el grupo doméstico de dońa Dina y su hija Adela, que viven juntas en la 
aldea, es un hogar extenso compuesto por el núcleo monoparental femenino de Adela (madre soltera) y sus dos hijas, dońa Dina (viuda) y el hijo primogénito de Rosa, "criado" por la abuela. El grupo de Rosa (madre soltera) y sus dos hijos menores, que viven en otra casa en la aldea, es un hogar nuclear monoparental femenino. Pero si miramos la casa de campo, cuando no hay escuela y todos se encuentran ahí para cuidar la hacienda, tendríamos un único grupo doméstico multinuclear femenino, compuesto por los dos núcleos monoparentales de Rosa y Adela con sus hijos, además de doña Dina. También el grupo doméstico de Marta y doña Ana es un hogar multinuclear femenino, compuesto por el núcleo monoparental de doña Ana (madre soltera), su hija menor y su sobrino "criado" por ella y el núcleo de Marta (madre soltera) y sus hijos, que comparten la casa de campo y la casa en la aldea. La tipología de familiahogares de Laslett (1972) nos resulta útil, pero hay que concebir dichas estructuras de manera dinámica y cambiante.

El concepto mismo de "casa" implica categorías y prácticas locales específicas. No solo hay diferentes casas, como la de campo, el puesto y la casa en la aldea, sino que "casa" puede corresponder tanto a la pieza-dormitorio, habitada por una persona sola, por una pareja con o sin hijos, por una madre soltera con hijos, como a todo el grupo de piezas (dormitorios y depósitos) con una cocina, ${ }^{12}$ pero también al conjunto residencial familiar en la aldea, que junta diferentes grupos de piezas y cocinas alrededor de patios comunes. Aunque doña Dina y Adela, por un lado, y Marta, por el otro, viven en la aldea en su casa aparte, los conjuntos habitacionales familiares son muy comunes. Se encuentran uno a lado del otro en la aldea y juntan varios grupos domésticos de una misma red familiar; por ejemplo los de padres e hijos o de hermanos y hermanas. En el conjunto habitacional familiar, cada gupo doméstico puede tener su "cocina aparte" y compartir con los demás espacios comunes, como los patios.

12 "Más allá de los usos que tengan cada uno de estos recintos, se les denomina casa, al igual que al conjunto general de recintos. Es decir la casa se compone de casas. La noción de casa hace referencia, entonces, tanto al todo como a la parte, entendiendo que esa parte se constituye también como una totalidad en sí misma" (Tomasi, 2011), en Bugallo y Tomasi, 2012, p. 212.
En muchas casas, además, hay una pieza para los Santos y las Virgencitas, o sea las estatuas e imágenes sagradas del grupo doméstico. Estas estatuas pueden tener un "dueño" individual, por lo general el "jefe” o "jefa” del hogar, ya que serían heredados por alguno de ellos, encontrados o comprados. Los Santos y Virgencitas son llevados en andas, atados uno a otros con cintas de colores, a la capilla de la aldea para las fiestas patronales, para Pascua y cuando llega el sacerdote desde la parroquia del valle para hacer misa y misionar. Las andas son llevadas en hombros a las procesiones y constituyen el elemento central del ritual de la "desatada", cuando los participantes desatan los Santitos y Virgencitas haciendo una ofrenda en dinero que será recompensada con dulces. Una desatada se realiza en las casas entre el 1 y el 2 de noviembre para las "almas".

En el altiplano de Jasimaná, además, "casa” implica la posibilidad de extensión material: según Tomasi $(2012$, p. $17 ; 2013$, pp. 78-79) se trata de una "construcción continua del espacio doméstico", ya que más piezas-dormitorios pueden ser agregadas (una al lado de la otra, formando una línea o una $\mathrm{L}$ o construidas alrededor del patio). Cada pieza es un espacio independiente con su propia puerta de salida hacia el patio. La casa de campo de Adela y dońa Dina en Cerro C., por ejemplo, está compuesta por la cocina y otra pieza al lado que pertenecían al padre de dońa Dina, y por otras dos piezas en frente que hizo construir doña Dina para sus hijas, además de la letrina un poco alejada, el horno y, sobre todo, los corrales para los animales.

Los que comparten la casa de campo, de hecho, comparten la hacienda también, es decir, tienen su propios animales juntos en la hacienda familiar cuidada por el pastor o pastora principal y sus ayudantes. En el estudio de caso, tanto el grupo de Marta y su madre Ana como el de Adela, dońa Dina y Rosa comparten la hacienda. Si bien solo doña Ana, en un caso, y Adela, en el otro, trabajan de pastoras principales cuidando los animales de los demás, los otros miembros de cada grupo les ayudan dándoles el cambio durante el fin de semana, colaborando en las actividades más pesadas como la esquila y participando en toda actividad ritual como el pago a la Tierra y las señaladas de los animales, verdaderas 
"tecnologías rituales de la producción" como lo han señalado Van Kessel (1992) y Bugallo (2014).

Por fin, como ya se subrayó, también los miembros de la familia que no viven en Jasimaná pueden tener sus animales en la hacienda familiar. Estos emigrantes regresan al altiplano para la esquila y para las señaladas. Durante estos trabajos rituales, cuando "señalan", los padres y los abuelos "nombran" unos animales jóvenes para sus hijos o nietos. Se trata de una "herencia" pero con vida, que se recibe de a poco, cuya salud y reproducción depende de la "suerte" individual de cada persona (Bugallo y Tomasi, 2012, p. 220). Estos animales que se reciben desde las niñez forman parte de la tropa individual, incorporada a la hacienda familiar. Cuando la persona se "separa" de sus padres para formar otro grupo doméstico en otra casa de campo se lleva sus animales.

Los pastores y pastoras reciben sus primeros animales por medio de un ritual llamado "ruti" (de la palabra quechua rutuy, cortar), cuando se corta por primera vez el pelo de un niño o de una niña (aproximadamente entre uno y tres años de edad). En esta ocasión los miembros de la red familiar se juntan y cada uno corta un mechón o una trencita llamada "simba" (de la palabra quechua sin'pa, trenza de cabellos) del pelo del nińo, haciendo una ofrenda "a voluntad". Generalmente se regala dinero, cuya cantidad los padres anotan, que sirve para comprar los primeros animales del niño. Por medio de este ritual los miembros adultos del grupo doméstico y de la red familiar del individuo crean la relación entre el futuro pastor o pastora y los animales de su hacienda, manejando un elemento especial, el cabello, que se considera guarda algo de la fuerza vital de una persona. $\mathrm{Al}$ respecto, se dice: "tenés que tener tu hacienda de tu pelo".

Otras prácticas rituales relacionadas con la hacienda son las seńaladas y el pago a la Tierra que se realiza en agosto, cuando se hace una ofrenda grande a la Pachamama-Santa Tierra, pidiendo salud para los miembros de la familia y para los animales. En las señaladas participan no solo los dueños de los animales, sino varios miembros de la familia y los vecinos también, mientras que en el "pago", que se realiza en la casa de campo generalmente el 1 de agosto, participan los que tienen sus animales juntos en la misma hacienda. En Ciénaga G., para el "pago" del 1 de agosto se juntaron doña Ana y sus hijos, entre ellos Marta con sus tres hijos, pero también una hermana del padre de dońa Ana, cuyos animales Ana "cuida" en la hacienda familiar.

Por lo tanto, más allá de la categoría "cocina aparte", la categoría "hacienda" y las prácticas conectadas nos ayudan a identificar al "grupo doméstico" como un grupo que comparte la casa de campo y la hacienda. Pero la "hacienda" y las prácticas relativas a ésta incluyen también a miembros de la familia que no comparten la casa de campo, sino solo ciertas actividades productivas y rituales relacionadas con los animales. Estas actividades, así como las prácticas habitacionales basadas en los conjuntos habitacionales familiares en la aldea, implican un ámbito de relaciones que se extiende desde el "grupo doméstico" hacia la "red familiar".

\section{"Ser familia" y "criar"}

Los que comparten la hacienda, generalmente, "son familia” entre sí. Esta categoría émica corresponde a la categoría ética "parentesco", ya que quienes son familia, son parientes, o sea, como se dice en Jasimaná, "se reconocen" en tanto que parientes y se respetan entre ellos. En cambio, los que son llamados "parientes" no son familia, es decir, no son parientes a nivel émico: no se reconocen ni respetan en tanto que parientes. Se dice, por ejemplo: "con los parientes ya no nos reconocemos, no somos familia". "Reconocerse" significa conocer la relación y actuar en consecuencia.

En este caso el parentesco no se basa tanto y solo en una sustancia biogenética compartida, como la "sangre", ${ }^{13}$ según la lógica del parentesco euroamericano (Schneider, 1968) que funda el concepto genealógico antropológico de parentesco. En la teoría local, "parentesco" se basa más en comportamientos. Se trata de "respetarse", lo que correspondería a la

13 Actualizando la idea de Schneider (1968), Solinas (2015) subraya que al concepto de "sangre" se puede sobreponer al concepto de ADN que, más bien que una "sustancia compartida", es un lenguaje, un código "biogenético" que cada vez se recombina. 
"conducta moral" que también define el parentesco euroamericano según Schneider, pero sobre todo se trata de "reconocerse", lo que implica una acción voluntaria.

Los que se reconocen como parientes, es decir, los que "son familia" entre sí, corresponden a los miembros del kindred ego-centrado y pueden ser identificados por medio del proceso de reconstrucción gráfica de las redes familiares, como en el estudio de caso. Estas redes, por lo general, no son muy extensas y llegan a incluir a los abuelos de ego, los padres, los hijos, los nietos, los hermanos de los padres, los hermanos y sus hijos, los primos hermanos y sus hijos. En nuestro estudio de caso, tanto Adela como Marta (primas segundas entre ellas) llegaron a extender su red familiar hasta incluirse respectivamente en tanto que primas segundas solo cuando yo les pregunté al respecto. En este caso la "memoria genealógica" (categoría antropológica) de las dos incluye la bisabuela común. Se trata de la pastora madre soltera que dejó a sus dos hijos (la abuela materna de Marta y el abuelo materno de Adela) los derechos de uso de los pastizales de Ciénaga G., por un lado, y de Cerro C. por el otro, que hoy en día garantizan la subsistencia de sus descendientes en Jasimaná. Desde que los confines de los pastizales de cada grupo doméstico dependen del principio del "respeto" de los territorios familiares, la memoria genealógica individual y familiar llega por lo menos hasta incluir a los antepasados (los padres, abuelos o bisabuelos) que dejaron a sus descendientes los derechos sobre las tierras. Todos los demás ancestros confluyen en la categoría émica de "los de antes": una categoría indiferenciada por grado genealógico y género que llega a incorporar a los antiguos habitantes del lugar, también llamados "los indios".

"Los de antes", los antepasados difuntos, vuelven a sus casas por el "día de las almas" entre el 1 y el 2 de noviembre. Se dice que en esta fecha las almas "vuelven adonde sabían vivir" y por esto se les espera preparando una "mesa" ritual con muchos platos de comida y con bebidas, cigarillos y hojas de coca. La mitad de todos estos bienes serán enterrados al mediodía del 2 de noviembre. Las "almas" son los antepasados, pero también los difuntos en general. Además, la categoría émica "alma" incluye tanto a seres muertos como a vivos, ya que cada persona tiene un "alma" que corres- ponde a su conciencia individual y está asociada a su nombre. Este alma individual se puede "perder" por un "susto"; en este caso tiene que ser "llamada" por un médico campesino para que vuelva. La noche del 1 de noviembre las almas de los abuelos y bisabuelos y las de los otros miembros de la familia fallecidos son "nombradas", es decir, que se leen sus nombres en las "intenciones" escritas por los familiares, después de haber realizado la "desatada" de los Santos y Virgencitas de las andas. Solo los "angelitos", los niños y niñas muertos con pocos meses o pocos ańos, no son nombrados. Se trata de una categoría sin género ni nombre, pero las personas recuerdan en el día de "las almas" a sus hijos angelitos.

Los antepasados, por lo tanto, están asociados a las casas. También se usa la categoría "sangre" para referirse a lo que se trasmite y mezcla por filiación y que se expresa en las actitudes personales "heredadas" de los antepasados ("tiene la mala sangre de su abuelo", etc.). A pesar de esto, lo que produce filiación a nivel local son sobre todo las prácticas conectadas a la categoría "criar", que implica hacer crecer, alimentar , "hacerse cargo" de una guagua (bebé) o de un niño. Quien cría puede ser padre y/o madre, pero también puede tratarse de una abuela o abuelo, de una tía o de alguien sin lazos de sangre con el niño o la nińa. El "criado" o la "criada" vive con su madre y/o padre de crianza, trabaja para él o ella, de quienes recibe sus animales. "Criar" se refiere a las plantas y a los animales también. Los habitantes de Jasimaná se definen "criadores" de la hacienda y hablan de sus animalitos con mucho cariño y ternura, dándoles nombres asociados al color del vello o a otras características. "Criar" tanto a los niños como la hacienda implica un proceso largo de prácticas diarias, laborales y rituales también.

En nuestro estudio de caso, el sobrino de doña Ana "se crió con" ella. El hijo primogénito de Rosa "se crió con" dońa Dina, su abuela. Algunos de los "criados" son "regalados" por padres que no pueden "hacerse cargo" de ellos, pero la mayoría son "hijos de soltera" que han sido dejados con los abuelos maternos o paternos cuando la mamá formó otro hogar con una nueva pareja. Los que se criaron con sus abuelos usan los términos de dirección "mami" y "papi" para los abuelos, pese a que muchas veces se refieren a ellos llamandoles "mi abuelita" o "mi 
abuelito" con otra persona. Los chicos criados por su propia madre o su proprio padre que se "junta", formando un "grupo doméstico recompuesto" (categoría antropológica), se vuelven "entenados" de la nueva pareja del progenitor.

En general, tener uno o más "hijos de soltera" es una práctica común tanto para las mujeres que luego se "juntan" en pareja, formando un nuevo hogar nuclear conyugal o extenso o multinuclear, como para las que siguen solteras en la casa de su madre o de sus padres o en el complejo residencial familiar de éstos y de sus hermanos.

El estudio de caso muestra la importancia de las madres solteras y de los grupos monoparentales femeninos en el altiplano de Jasimaná. Sin embargo, existen expresiones locales como "quien se casa quiere casa" que valoran la pareja y la formación de un hogar nuclear conyugal, aunque por lo general las parejas "se juntan" y no se casan tan frecuentemente. En este caso se "separan" de la casa de sus padres o de su madre y van a vivir "aparte".

La mayoría de los hombres adultos del altiplano forman una pareja estable con su mujer e hijos, pero pueden tener otros hijos "aparte" con diferentes mujeres solteras que viven solas con ellos o con sus padres, abuelos y hermanos. Una mujer soltera, además, puede tener hijos con hombres diferentes. Pese a que muchos de estos hombres-padres no se hacen cargo de sus hijos naturales, nacidos fuera de una relación estable o matrimonio, pueden "reconocerlos" y también ayudarles de alguna manera. "Reconocer" no implica un acto legal de reconocimiento, sino aceptar socialmente el lazo de parentesco, el "ser familia". Para un hombre-padre, reconocer a sus hijos naturales es fuente de cierto orgullo y hay expresiones locales que conectan la riqueza y la hacienda a una prole numerosa: "muchos hijos, mucha hacienda" se dice.

Cuando un padre natural no "reconoce", en tanto que "familia", a su hijo natural, o también cuando la madre no "hace reconocer" el padre natural al hijo que tuvo de soltera, se puede decir del niño que es "hijo o hija del viento", ya que en muchas expresiones locales, sobre todo en los chistes y en las coplas, el hombre-padre que desaparece es descrito como un "vientito" que llegó y se fue (Salvucci, 2015).

\section{Culturas locales del parentesco}

El estudio de caso presentado nos permite afirmar que las categorías antropológicas no siempre logran explicar las situaciones concretas y que los conceptos de "grupo doméstico" y "red familiar" solo tienen sentido en una perspectiva de traducción, como punto de partida para acercarnos a comprender las relaciones locales y las categorías émicas. Éstas pueden tanto exceder las nociones antropológicas como compactarlas en un único concepto de convergencia.

Con respecto a los "grupos domésticos" de Jasimaná, hay que subrayar que los grupos concretos no solo se transforman en el tiempo, ${ }^{14}$ sino que también se modifican en el espacio, según los circuitos de movimiento de sus miembros. Los hogares representados en forma gráfica en el estudio de caso no son estructuras fijas, sino dinámicas: sus miembros se desplazan constantemente entre diferentes viviendas, moviéndose a lo largo del altiplano en los circuitos de la trashumancia y entre el altiplano y el valle en circuitos comerciales y migratorios. Además, todos los integrantes del grupo se mueven entre la casa en la aldea y la casa principal (o el puesto, según la temporada) para turnarse en el cuidado de los animales que comparten. Este patrón de "residencia múltiple” (Gil Montero, 2004) y los circuitos de movimiento asociados a las prácticas del pastoreo, a la escuela, a los viajes de comercio y venta de productos en los mercados de los valles complican el concepto de "estructura de familia-hogar" (Laslett, 1972), ya que sus miembros no viven siempre "bajo un mismo techo", sino que se desparraman, se desplazan, circulan de una casa a otra, viven al mismo tiempo en casas diversas, se turnan, se juntan y se separan. Se trata de grupos domésticos "elásticos" que se agregan y disgregan.

14 Agrandándose y reduciéndose según incorporen una nueva pareja conyugal o una hija madre soltera con sus hijos, o que pierdan a sus integrantes emigrados de forma temporánea o permanente. Además, las parejas se juntan y se separan; los hijos son dejados a los abuelos para que los críen pero también pueden ser reclamados y volver con sus padres o con la madre y su nueva pareja. 
El análisis de estas prácticas y de las categorías émicas performadas y utilizadas en Jasimaná permite destacar ciertas características culturales del parentesco local.

Las categorías y prácticas en las cuales nos detuvimos se centran en la "cocina", que se comparte o que se tiene "aparte", y en la "casa", que es tanto la "de campo" como la de la aldea. La categoría émica "casa", además, se refiere a la pieza, al grupo de piezas con cocina, pero también al conjunto habitacional familiar con patios comunes, lo que hace posible interpretar al "grupo doméstico" local como un ámbito de relaciones extensible. Este ámbito relacional se basa tanto en las prácticas habitacionales como en las prácticas centradas en la "hacienda". El trabajo pastoril, los circuitos de la trashumancia, los turnos en el cuidado de los animales y la colaboración entre la pastora principal y sus ayudantes, pero también las prácticas rituales como la ruti, el pago a la Tierra y las señaladas, producen un ámbito de relaciones en el que "hacienda" y "familia" convergen, pese a que hay actividades, como por ejemplo las señaladas, en las cuales participan también los vecinos que no son familia.

Las relaciones centradas en la casa y en la hacienda, además, no solo incluyen a las personas y sus animales, sino también a entidades como los Santos y las Virgencitas que habitan en una pieza de la casa y a los antepasados que vuelven en tanto que "almas" donde "sabían vivir". Los que comparten la casa y la hacienda, por fin, por lo general "son familia" entre sí. "Ser familia" corresponde a la categoría local de parentesco e implica prácticas como "reconocerse" y "respetarse", que conllevan acciones y comportamientos para actuar, más que "esencias" o "sustancias" transmisibles de forma genealógica.

Otra categoría émica que expresa las relaciones de parentesco en tanto que filiación es "criar", que implica un proceso largo de actividades diarias y acciones laborales y rituales. La relación entre quien cría y quien es criado no depende de la transmisión de una sustancia biogenética ni de una adopción jurídicosocial, sino del "cuidado" y del "hacerse cargo". La misma categoría expresa las relaciones entre los pastores y sus animales. La persona "criada" puede ser un hijo, un nieto o un sobrino o no tener lazos de consanguinidad con sus padres de crianza, aunque la categoría émica específica "criado" o "criada" indica un hijo ajeno.

Las prácticas del "criar" hijos ajenos se basan en cierta "circulación" de los hijos, sobre todo de los hijos de solteras que pueden ser dejados con los abuelos o llevados por su mamá a la casa de su nueva pareja.

La práctica del tener "hijos de soltera" junto a la de "criar" fundamentan un universo local de parentesco fluido, basado en la circulación de los hijos y de los hombres-padres y en la autonomía reproductiva femenina, a pesar de la valorización de la "pareja".

Por fin, analizando las características del parentesco local en relación a las pautas culturales difundidas a lo largo de la macro área andina, el alcance histórico-geográfico macrorregional de dichas prácticas y categorías resulta evidente. En Jasimaná, el "sistema" de parentesco es bilateral, según el esquema del kindred de Freeman (1961), y la devolución incluye a todos los hijos, varones y mujeres, según el modelo de Lambert (1980 [1977]). La pareja conyugal juega un rol importante, aunque la práctica de tener hijos de soltera y la centralidad de las "madres solteras" en el universo local de las relacionas indican la presencia de características matrifocales, lo que también ocurre en otras comunidades a lo largo de la macro área andina. Por fin, desde una perspectiva culturalista, tanto en Jasimaná como en otras comunidades de la macro área andina (Yanagisako, 1979; Weismantel, 1988; Arnold, 1998; Carsten, 2000), el ámbito del parentesco se fundamenta en procesos y acciones, como las conectadas a la "cocina", a la "casa", al "criar" y al "reconocerse".

\section{Referencias citadas}

Arnold, D. (Comp.). (1997). Más allá del silencio: las fronteras de género en los Andes. Tomo I, La Paz: ILCA/CIASE.

Arnold, D., (1998). Introducción. De “castas” a kastas. Enfoques hacia el parentesco andino. En Gente de carne y hueso: las tramas de parentesco en los Andes (pp. 15-66). La Paz: ILCA/CIASE.

Arnold, D., Jiménez, D., y Yapita, J. D. D., (1998a). Hacia un Orden Andino de las Cosas, La Paz: HISBOL/ILCA. 
Assadourian, C. S. (1982). El sistema de la economía colonial. Mercado interno, regiones y espacio económico. Lima: IEP.

Belote, J., y Belote, L. (1980 [1977]). Parentesco y limitación de obligaciones en Saraguro. En Mayer, E. y Bolton, R. (Comps.). Parentesco y matrimonio en los Andes (pp. 463-479). Lima: Pontificia Universidad Católica del Perú.

Brush, S. B. (1977). Mountain, Field and Family: The economy and Human Ecology of an Andean Valley. Pennsylvania: University of Pennsylvania Press.

Bugallo, L. (2014). Flores para el ganado. Una concepción puneña del multiplico. (Puna de Jujuy, Argentina). En Rivera Andía, J. J. (Comp.). Comprender los rituales ganaderos en los Andes y más allá. Etnografías de lidias, herranzas y arrierias (pp. 311-363). Aachen: Schaker Verlag.

Bugallo, L., y Tomasi, J. (2012). Crianzas mutuas. El trato a los animales desde las concepciones de los pastores puneños (Jujuy, Argentina). Revista Española de Antropología Americana, 42(1), 205-224.

Carrasco, A. M., y González, H. (2014). Movilidad poblacional y procesos de articulación rural-urbano entre los aymara del norte de Chile. Si Somos Americanos. Revista de Estudios Transfronterizos, XIV(2), 217-231.

Carsten, J. (Comp.). (2000). Cultures of Relatedness. New Approaches to the Study of Kinship. Cambridge: Cambridge University Press.

Castro Pozo, H. (1946). Social and economico-political evolution of the communities in Central Peru. En Steward, J. E. (Ed.). Handbook of the South American Indians. The Andean Civilizations, vol. 2 (pp. 483-499). Washington: USA Government Printing Office.

De La Cadena, M. (1988). Comuneros en Huancayo. Migración campesina a ciudades serranas. Lima: IEP.

De La Peña, G. (2001). Los desafíos de la clase incómoda: el campesinado frente a la antropología americanista. En León-Portilla, M. (Comp.). Motivos de la antropología americanista. Indagaciones en la diferencia (pp. 134-166). México: Fondo de Cultura Económica.

Delfino, D. (2001). Of Pircas and the Limits of Society: Ethnoarchaeology in the Puna, Laguna Blanca, Catamarca, Argentina. En Kuznar, L. (Ed.), Ethnoarchaeo- logy of Andean South America (pp. 97-137). Michigan: International Monographs in Prehistory.

Freeman, J. (1961). On the Concept of Kindred. The Journal of the Royal Anthropological Institute of Great Britain and Ireland XCI(2), 192-220.

Geertz, C. (1973). The Interpretation of Cultures. New York: Basic Books.

Gil Montero, R. (2004). Caravaneros y trashumantes en los Andes Meridionales. Población y familia indigena en la puna de Jujuy 1770-1870. Lima: IEP.

Göbel, B. (2002). La arquitectura del pastoreo: uso del espacio y sistema de asentamiento en la Puna de Atacama (Susques). Estudios Atacameños, 23, 53-76.

Harris, M. (1968). The Rise of Anthropological Theory. London: Routledge \& Kegan Paul.

Harris, O. (1985). Ecological Duality and Role of the Center: Northen Potosí. En Shozo, M., Izumi, S., and Craig, M. (Comps.). Andean Ecology and civilization. An Interdisciplinary Perspective on Andean Ecological Complementary (pp. 311-335). Tokyo: University of Tokyo Press.

Hidalgo, J. (1985). Ecological complementarity and tribute in Atacama: 1863-1792. En Masuda, S., Shimada, I., Morris, C. (Comps.). Andean Ecology and Civilitations (pp. 161-184). Tokyo: University of Tokyo Press.

Isbell, B. J. (2005 [1978]). Para defendernos. Ecología y ritual en un pueblo andino. Cuzco: Centro Bartolomé de Las Casas.

Lambert, B. (1980 [1977]). Bilateralidad en los Andes. En Mayer, E., y Bolton, R. (Comps.). Parentesco y matrimonio en los Andes (pp. 11-54). Lima: Pontificia Universidad Católica del Perú.

Laslett, P. (1972). La famille et le ménage. Annales Économies, Sociétés, Civilisations, 847-872.

Lévi-Strauss, C. (1967 [1949]). Les structures élémentaires de la parenté. Paris: Mouton.

Lorandi, A. M. (1980). La frontera oriental del Tawantinsuyo: el Umasuyo y el Tucumán. Una hipótesis de trabajo. Relaciones de la Sociedad Argentina de Antropología, XIV(1), 147-164. 
Lorandi, A. M. (1990-92). Ni tradición ni modernidad. El mestizaje en contextos sociales desestructurados. Relaciones de la Sociedad Argentina de Antropología, XVIII, 93-120.

Lorandi, A. M. (Comp.). (1997). El Tucumán Colonial y Charcas. Tomo I. Buenos Aires: Facultad de Filosofía y Letras, UBA.

Lorandi, A. M. (1997a). De quimeras, rebeliones y utopias. La gesta del inca Pedro Bohorques. Lima: Pontificia Universidad Católica del Perú.

Malengreau, J. (2007). Migraciones entre lo local y lo regional en los Andes peruanos: redes rural-urbanas, fragmentaciones espaciales y recomposiciones identitarias. Bulletin IFEA Institut Français d'Etudes Andines, 36(3), 427-445.

Mata De López, S. (2005). Tierra y poder en Salta. El noroeste argentino en vísperas de la Independencia. Salta: CEPIHA.

Mayer, E. (2004). Casa, chacra y dinero. Economias domésticas y ecología en los Andes. Lima: IEP.

Mayer, E., y Bolton, R. (Comps.). (1980). Parentesco y matrimonio en los Andes. Lima: Pontificia Universidad Católica del Perú.

Mishkin, B. (1946). The Contemporary Quechua. En Steward, J. E. (Ed.). Handbook of the South American Indians. The Andean Civilizations, vol. 2 (pp. 409-499). Washington: USA Government Printing Office.

Ossio, J. M. (1982). Estructura social y parentesco en la antropologia sobre el area andina. Anuario Antropológico, $80,223-252$.

Palacios Ríos, F. (1982). El simbolismo aymara de la casa. Boletín del Instituto de Estudios Aymaras, 2(12), 37-57.

Parodi, M., y Benedetti, A. (2016). Minería, descampesinización y desocupación. Trayectorias de movilidad de cuatro mineros de El Aguilar (Jujuy, Argentina, dácada de 1940 a 2010). Estudios Atacameños. Arqueología y antropología surandinas, 52, 25-48.

Piasere, L., y Solinas, P. G. (1998). Le culture della parentela e l'esogamia perfetta. Roma: CISU.
Pike, K. L. (1967 [1954]). Language in relation to a Unified Theory of the structure of Human Behavior. Paris: Muton $\&$ Co.

Platt, T. (1978). Symétries en miroir. Le concept de yanantin chez les Macha de Bolivie. Annales, Économies, Sociétés, Civilisations, 33(5-6), 1081-1107.

Rabey, M., Merlino, R., y González, D. (1986). Trueque, articulación económica y racionalidad campesina en el Sur de los Andes Centrales. Revista Andina, 1(4), 131-160.

Reboratti, C. (1976). Migración estacional en el Noroeste Argentino y su repercusión en la estructura agraria. Demografía y Economía, X(2), 235-253.

Rodríguez, L. B., y Lorandi, A. M. (2005). Apropiaciones y usos del pasado. Historia y patrimonio en el Valle Calchaquí. Bulletin de l'Institut Français d'Études Andines, 34(3), 431-442.

Salvucci, D. (2010). Mappe genealogiche e romanzo di formazione. Kindred, rappresentazioni e pratiche parentali giovanili. En Grilli, S., y Zanotelli, F. (Comps..). Scelte di famiglia. Tendenze della parentela nella società contemporanea (pp. 205-231). Pisa: Edizioni ETS.

Salvucci, D. (2015). Mujeres madres-solteras y circulación de hombres padres en el altiplano de Jasimaná, Salta, Argentina. En O. Guidobono y Caño Ortigosa (Comps.), Temas americanistas: historia y diversidad cultural (pp. 705-714). Sevilla: Editorial Universidad de Sevilla.

Sánchez-Albornoz, N. (1978). Indio y tributo en el Alto Perú. Lima: IEP.

Schneider, D. M. (1968). American Kinship: A Cultural Account. Chicago: University of Chicago Press.

Schneider, D. M. (1984). A Critique of the Study of Kinship. USA: University of Michigan.

Sendón, P. (2005). Ecología, ritual y parentesco en los Andes. Notas a un debate no permitido. Debate Agrario, 4041, 273-297.

Sinibaldi, S. (2008). La dimensione spazio- temporale degli aggregati domestici. Riflessioni su famiglia, parentela e residenza in un comune toscano. En P. P. Viazzo y A. Rosina A. (Comps.), Oltre le mura domestiche. Famiglia 
e legami intergenerazionali dall'Unità d'Italia ad oggi (pp. 171-202). Udine: Forum.

Solinas, P. G. (2015). Ancestry. Parentele elettroniche e lignaggi genetici. Firenze: EditPress.

Spedding, A. P. (1998). Contra-afinidad: algunos comentarios sobre el compadrazgo andino. En Arnold, D. (Comp.). Gente de carne y hueso: las tramas de parentesco en los Andes. Tomo II (pp. 115-138). La Paz: ILCA/ CIASE.

Stone, L. (Comp.). (2001). New Directions in Anthropological Kinship. USA: Rowman \& Littlefield Publishers.

Tomasi, J. (2012). Lo cotidiano, lo social y lo ritual en la práctica del construir. Aproximaciones desde la arquitectura puneña (Susques, provincia de Jujuy, Argentina). Apuntes, 25(1), 8-21.

Tomasi, J. (2013). Espacialidades pastoriles en las tierras altoandinas. Asentamientos y movilidades en Susques, puna de Atacama (Jujuy, Argentina). Revista de Geografía Norte Grande, 55, 67-87.

Tschopik, H. (1946). The Aymara. En Steward, J. E. (Comp.). Handbook of the South American Indians. The Andean Civilizations, vol. 2 (pp. 501-573). Washington: USA Government Printing Office.
Van Kessel, J. (1992). Tecnología aymara: un enfoque cultural. En Van den Berg, H. (Comp.). Cosmovisión aymara (pp. 187-220). La Paz: UCB/HISBOL.

Vásquez, M., Homberg, A. (1966). The Castas: Unilineal Kin Groups in Vico, Perú. Ethnology, 5, 284-303.

Weismantel, M. (1988). Food, Gender and Poverty in the Ecuadrian Andes. Philadelphia: University of Pennsylvania Press.

Weismantel, M. (1998). Viñachina: hacer guaguas en Zumbagua, Ecuador. En Denise, A. (Comp.). Gente de carne $y$ hueso: las tramas de parentesco en los Andes. Tomo II (pp. 83-95). La Paz: ILCA/CIASE.

Wolf, E. R. (1966). Peasants. New Jersey: Prentice-Hall, Englewood Cliffs.

Yanagisako, S. J. (1979). Family and Household: The Analysis of Domestic Groups. Annual Review of Anthropology, 8, 161-205. 